\title{
ON TREE IDEALS
}

\section{MARTIN GOLDSTERN, MIROSLAV REPICKÝ, SAHARON SHELAH, AND OTMAR SPINAS}

(Communicated by Andreas R. Blass)

\begin{abstract}
Let $l^{0}$ and $m^{0}$ be the ideals associated with Laver and Miller forcing, respectively. We show that $\operatorname{add}\left(l^{0}\right)<\operatorname{cov}\left(l^{0}\right)$ and $\operatorname{add}\left(m^{0}\right)<\operatorname{cov}\left(m^{0}\right)$ are consistent. We also show that both Laver and Miller forcing collapse the continuum to a cardinal $\leq \mathfrak{h}$.
\end{abstract}

\section{INTRODUCTION AND NOTATION}

In this paper we investigate the ideals connected with the classical tree forcings introduced by Laver [La] and Miller [Mi]. Laver forcing $\mathbb{L}$ is the set of all trees $p$ on ${ }^{<\omega} \omega$ such that $p$ has a stem and whenever $s \in p$ extends $\operatorname{stem}(p)$ then $\operatorname{Succ}_{p}(s):=\left\{n: s^{\wedge} n \in p\right\}$ is infinite. Miller forcing $\mathbb{M}$ is the set of all trees $p$ on ${ }^{<\omega} \omega$ such that $p$ has a stem and for every $s \in p$ there is $t \in p$ extending $s$ such that $\operatorname{Succ}_{p}(t)$ is infinite. We denote the set of all these splitting nodes in $p$ by $\operatorname{Split}(p)$. For any $t \in \operatorname{Split}(p), \operatorname{Split}_{p}(t)$ is the set of all minimal (with respect to extension) members of $\operatorname{Split}(p)$ which properly extend $t$. For both $\mathbb{L}$ and $\mathbb{M}$ the order is inclusion.

The Laver ideal $l^{0}$ is the set of all $X \subseteq{ }^{\omega} \omega$ with the property that for every $p \in \mathbb{L}$ there is $q \in \mathbb{L}$ extending $p$ such that $X \cap[q]=\varnothing$. Here $[q]$ denotes the set of all branches of $q$. The Miller ideal $m^{0}$ is defined analogously, using conditions in $\mathbb{M}$ instead of $\mathbb{L}$. By a fusion argument one easily shows that $l^{0}$ and $m^{0}$ are $\sigma$-ideals.

The additivity (add) of any ideal is defined as the minimal cardinality of a family of sets belonging to the ideal whose union does not. The covering number (cov) is defined as the least cardinality of a family of sets from the ideal whose union is the whole set on which the ideal is defined- ${ }^{\omega} \omega$ in our case. Clearly $\omega_{1} \leq \operatorname{add}\left(l^{0}\right) \leq \operatorname{cov}\left(l^{0}\right) \leq \mathfrak{c}$ and $\omega_{1} \leq \operatorname{add}\left(m^{0}\right) \leq \operatorname{cov}\left(m^{0}\right) \leq \mathfrak{c}$ hold.

Received by the editors January 22, 1993 and, in revised form, August 9, 1993.

1991 Mathematics Subject Classification. Primary 03E35, 03E50, 54A25.

The first and third authors were supported by DFG grant Ko 490/7-1, and by the Edmund Landau Center for research in Mathematical Analysis, supported by the Minerva Foundation (Germany). Publication 487.

The second author was supported by the Basic Research Foundation of the Israel Academy of Sciences and by grant GA SAV 365 of the Slovak Academy of Sciences.

The fourth author was supported by the Basic Research Foundation of the Israel Academy of Sciences and the Schweizer Nationalfonds. 
The main result in this paper says that there is a model of ZFC where $\operatorname{add}\left(l^{0}\right)<\operatorname{cov}\left(l^{0}\right)$ and $\operatorname{add}\left(m^{0}\right)<\operatorname{cov}\left(m^{0}\right)$ hold. The motivation was that by a result of Plewik [P1] it was known that the additivity and the covering number of the ideal connected with Mathias forcing are the same and they are equal to the cardinal invariant $\mathfrak{h}$-the least cardinality of a family of maximal antichains of $\mathscr{P}(\omega) / f$ in without a common refinement. On the other hand, in [JuMiSh] it was shown that $\operatorname{add}\left(s^{0}\right)<\operatorname{cov}\left(s^{0}\right)$ is consistent, where $s^{0}$ is Marczewski's ideal-the ideal connected with Sacks forcing $\mathbb{S}$. Intuitively, $\mathbb{L}$ and $\mathbb{M}$ sit somewhere between Mathias forcing and $\mathbb{S}$. In [GoJoSp] it was shown that under Martin's axiom $\operatorname{add}\left(l^{0}\right)=\operatorname{add}\left(m^{0}\right)=\mathfrak{c}$, whereas this is false for $s^{0}$ (see [JuMiSh]).

The method of proof for $\operatorname{add}\left(s^{0}\right)<\operatorname{cov}\left(s^{0}\right)$ in [JuMiSh] is the following: For a forcing $P$ denote by $\kappa(P)$ the least cardinal to which forcing with $P$ collapses the continuum. In [JuMiSh] it is shown that $\operatorname{add}\left(s^{0}\right) \leq \kappa(\mathbb{S})$. In [BaLa] it was shown that in $V^{\mathbf{S}_{\omega_{2}}} \kappa(\mathbb{S})=\omega_{1}$ holds, where $\mathbb{S}_{\omega_{2}}$ is the countable support iteration of length $\omega_{2}$ of $\mathbb{S}$. Hence $V^{\mathbf{S}_{\omega_{2}}} \models \operatorname{add}\left(s^{0}\right)=\omega_{1}$. On the other hand, a Löwenheim-Skolem argument shows that $V^{\mathbf{s}_{\omega_{2}}} \models \operatorname{cov}\left(s^{0}\right)=\omega_{2}$.

Our method of proof is similar. Denoting by $P_{\omega_{2}}$ a countable support iteration of length $\omega_{2}$ of $\mathbb{L}$ and $\mathbb{M}$ (each occurring on a stationary set), in $\S 2$ we prove the following:

\section{Theorem.}

$$
V^{P_{\omega_{2}}} \vDash \omega_{1}=\operatorname{add}\left(l^{0}\right)=\operatorname{add}\left(m^{0}\right)<\operatorname{cov}\left(l^{0}\right)=\operatorname{cov}\left(m^{0}\right)=\omega_{2} .
$$

The crucial steps in the proof are to show that $\kappa(\mathbb{L}), \kappa(\mathbb{M})$ equal $\omega_{1}$ and $\operatorname{add}\left(l^{0}\right) \leq \kappa(\mathbb{L}), \operatorname{add}\left(m^{0}\right) \leq \kappa(\mathbb{M})$ hold.

We will use the standard terminology for set theory and forcing. By $\mathfrak{b}$ we denote the least cardinality of a family of functions in ${ }^{\omega} \omega$ which is unbounded with respect to eventual dominance and $\mathfrak{d}$ will be the least cardinality of a dominating family in ${ }^{\omega} \omega$. Moreover, $\mathfrak{p}$ is the least cardinality of a filter base on $\left([\omega]^{\omega}, \subseteq^{*}\right)$ without any lower bound, and $t$ is the least cadinality of a decreasing chain in $\left([\omega]^{\omega}, \subseteq^{*}\right)$ without any lower bound. It is easy to see that $\omega_{1} \leq \mathfrak{p} \leq \mathfrak{t} \leq \mathfrak{b} \leq \mathfrak{d} \leq \mathfrak{c}$.

\section{UPPER AND LOWER BOUNDS}

Theorem 1.1. (1) $\mathfrak{t} \leq \operatorname{add}\left(l^{0}\right) \leq \operatorname{cov}\left(l^{0}\right) \leq \mathfrak{b}$.

(2) $\mathfrak{p} \leq \operatorname{add}\left(m^{0}\right) \leq \operatorname{cov}\left(m^{0}\right) \leq \mathfrak{d}$.

Proof of Theorem 1.1(1). We have to prove the first and the third inequality. For the third inequality, let $\left\langle f_{\alpha}: \alpha<\mathfrak{b}\right\rangle$ be an unbounded family. Define

$$
X_{\alpha}:=\left\{f \in{ }^{\omega} \omega:\left(\exists^{\infty} k\right) f(k)<f_{\alpha}(k)\right\} .
$$

Clearly $\bigcup\left\{X_{\alpha}: \alpha<\mathfrak{b}\right\}={ }^{\omega} \omega$. We claim $X_{\alpha} \in l^{0}$. Let $p \in \mathbb{L}$. We define $q \in \mathbb{L}$ as follows: $\operatorname{stem}(q):=\operatorname{stem}(p)$, and for any $s$ extending $\operatorname{stem}(q)$ we have $s \in q$ if and only if $s \in p$ and $(\forall k)$ if $|\operatorname{stem}(q)| \leq k<|s|$, then $s(k) \geq f_{\alpha}(k)$. Then clearly $q \in \mathbb{L}, q$ extends $p$, and $[q] \cap X_{\alpha}=\varnothing$.

In order to prove the first inequality we use the following notation from [JuMiSh]: Let $Q:=\left\{\bar{A}=\left\langle A_{s}: s \in{ }^{<\omega} \omega\right\rangle:(\forall s) A_{s} \in[\omega]^{\omega}\right\}$. For $\bar{A} \in Q$ we 
define a sequence of Laver trees $\left\langle p_{s}(\bar{A}): s \in{ }^{<\omega} \omega\right\rangle$ as follows: $p_{s}(\bar{A})$ is the unique Laver tree such that $\operatorname{stem}\left(p_{s}(\bar{A})\right)=s$ and if $t \in p_{s}(\bar{A})$ extends $s$, then $\operatorname{Succ}_{p_{s}(\bar{A})}(t)=A_{t}$.

For $\bar{A}, \bar{B} \in Q$ we define:

$$
\begin{gathered}
\bar{A} \subseteq \bar{B} \Leftrightarrow(\forall s) A_{s} \subseteq B_{s}, \\
\bar{A} \subseteq^{*} \bar{B} \Leftrightarrow(\forall s) A_{s} \subseteq^{*} B_{s}, \\
\bar{A} \leq^{*} \bar{B} \Leftrightarrow(\forall s) A_{s} \subseteq^{*} B_{s} \wedge\left(\forall^{\infty} s\right) A_{s} \subseteq B_{s} .
\end{gathered}
$$

Here $\leq^{*}$ is a slight but important modification of $\subseteq^{*}$ from [JuMiSh].

Fact 1.2. $\left(Q, \leq^{*}\right)$ is $\mathfrak{t}$-closed.

Proof of Fact 1.2. Suppose $\left\langle\bar{A}_{\alpha}: \alpha<\gamma\right\rangle$, where $\gamma<\mathfrak{t}$ is a decreasing sequence in $\left(Q, \leq^{*}\right)$. Let $\bar{A}_{\alpha}:=\left\langle A_{s}^{\alpha}: s \in{ }^{<\omega} \omega\right\rangle$. Since $\gamma<\mathfrak{t}$, there is $\bar{B}^{\prime}=\left\langle B_{s}^{\prime}\right.$ : $\left.s \in{ }^{<\omega} \omega\right\rangle \in Q$ such that $(\forall \alpha<\gamma) \bar{B}^{\prime} \subseteq^{*} \bar{A}_{\alpha}$. Define $f_{\alpha}:{ }^{<\omega} \omega \rightarrow \omega$ such that $(\forall s) B_{s}^{\prime} \backslash f_{s}(\alpha) \subseteq A_{s}^{\alpha}$. Since $\mathfrak{t} \leq \mathfrak{b}$, there exists $f:{ }^{<\omega} \omega \rightarrow \omega$ such that $(\forall \alpha)\left(\forall^{\infty} s\right) f_{\alpha}(s) \leq f(s)$. Now let $B_{s}:=B_{s}^{\prime} \backslash f(s)$ and $\bar{B}:=\left\langle B_{s}: s \in{ }^{<\omega} \omega\right\rangle$. It is easy to check that $(\forall \alpha<\gamma) \bar{B} \leq * \bar{A}_{\alpha}$.

Fact 1.3. Suppose $X \in l^{0}$ and $\bar{A} \in Q$. There exists $\bar{B} \in Q$ such that $\bar{B} \subseteq \bar{A}$ and $\left(\forall s \in{ }^{<\omega} \omega\right)\left[p_{s}(\bar{B})\right] \cap X=\varnothing$.

Proof of Fact 1.3. First note that if $D:=\{p \in \mathbb{L}:[p] \cap X=\varnothing\}$, then $D$ is open dense and even 0 -dense, i.e., for every $p \in \mathbb{L}$ there exists $q \in D$ extending $p$ such that $\operatorname{ste} m(q)=\operatorname{stem}(p)$. The proof of this is similar to Laver's proof in [La] that the set of Laver trees deciding a sentence in the language of forcing with $\mathbb{L}$ is 0 -dense: Suppose $p \in \mathbb{L}$ has no 0 -extension whose branches are not in $X$. Then inductively we can construct $q \in \mathbb{L}$ extending $p$ such that every extension of $q$ has a branch in $X$, contradicting $X \in l^{0}$.

Using this it is straightforward to construct $\bar{B}$ as desired.

Fact 1.4. Suppose $X \subseteq{ }^{\omega} \omega, \bar{A}, \bar{B} \in Q, \bar{B} \leq^{*} \bar{A}$, and $(\forall s)\left[p_{s}(\bar{A})\right] \cap X=\varnothing$. Then $(\forall s)\left[p_{s}(\bar{B})\right] \cap X=\varnothing$.

Proof of Fact 1.4. Clearly, if $F \subseteq p_{s}(\bar{B})$ is finite, then

$$
\left[p_{s}(\bar{B})\right]=\bigcup\left\{\left[p_{t}(\bar{B})\right]: t \in p_{s}(\bar{B}) \backslash F\right\} .
$$

But for almost all $t \in p_{s}(\bar{B}), p_{t}(\bar{B})$ extends $p_{t}(\bar{A})$. So clearly $\left[p_{s}(\bar{B})\right] \subseteq\left[p_{s}(\bar{A})\right]$ and hence $\left[p_{s}(\bar{B})\right] \cap X=\varnothing$.

End of the proof of Theorem 1.1(1). Suppose we are given $\left\langle X_{\alpha}: \alpha\langle\gamma\rangle\right.$ and $q \in \mathbb{L}$, where $\gamma<\mathfrak{t}$ and $(\forall \alpha) X_{\alpha} \in l^{0}$. Choose $\bar{A} \in Q$ such that $p_{\text {stem }(q)}(\bar{A})=q$, and let $\bar{B}_{0}$ be the $\bar{B}$ given by Fact 1.3 for $\bar{A}$ and $X_{0}$. If $\left\langle\bar{B}_{\alpha}: \alpha<\beta\right\rangle$ is constructed for $\beta \leq \gamma$ and $\beta$ is a successor, then choose $\bar{B}_{\beta}$ as given by Fact 1.3 for $\bar{A}=\bar{B}_{\beta-1}$ and $X=X_{\beta}$. If $\beta$ is a limit, then by Fact 1.2 choose first $\bar{A}$ such that $(\forall \alpha<\beta) \bar{A} \leq^{*} \bar{B}_{\alpha}$ and then find $\bar{B}_{\beta} \subseteq \bar{A}$ as given by Fact 1.3 for $\bar{A}$ and $X=X_{\beta}$. Finally, if we have constructed $\bar{B}_{\gamma}=\left\langle B_{s}^{\gamma}: s \in{ }^{<\omega} \omega\right\rangle$, define $\bar{B}:=\left\langle B_{s}: s \in{ }^{<\omega} \omega\right\rangle$ by $B_{s}:=B_{s}^{\gamma} \cap \operatorname{Succ}(s)$ if $s \in q$ extends $\operatorname{ste} m(q)$, and $B_{s}:=B_{s}^{\gamma}$ otherwise. It is easy to check that $\bar{B} \in Q, p_{\text {stem }(q)}(\bar{B})$ extends $q$ and $(\forall \alpha<\gamma)\left[p_{\text {stem }(q)}(\bar{B})\right] \cap X_{\alpha}=\varnothing$. 
Proof of Theorem 1.1(2). The proof is similar to (1). For the third inequality, let $\left\langle f_{\alpha}: \alpha<\mathfrak{d}\right\rangle$ be a dominating family. Define

$$
X_{\alpha}:=\left\{f \in{ }^{\omega} \omega:\left(\forall^{\infty} k\right) f(k)<f_{\alpha}(k)\right\} .
$$

Then $\bigcup\left\{X_{\alpha}: \alpha<\mathfrak{d}\right\}={ }^{\omega} \omega$ and in an analogous way as in (1) it can be seen that $X_{\alpha} \in m^{0}$.

In order to prove the first inequality we need the following concept from [GoJoSp]. Let $R$ be the set of all $\bar{P}=\left\langle P_{s}: s \in{ }^{<\omega} \omega\right\rangle$ where each $P_{s} \subseteq{ }^{<\omega} \omega$ is infinite, $t \in P_{s}$ implies $s \subset t$, and if $t, t^{\prime} \in P_{s}$ are distinct, then $t(|s|) \neq t^{\prime}(|s|)$. Given $\bar{P} \in R$ we can define $\left\langle p_{s}(\bar{P}): s \in \in^{<\omega} \omega\right\rangle$ as follows: $p_{s}(\bar{P})$ is the unique Miller tree with stem $s$ such that if $t \in \operatorname{Split}\left(p_{s}(\bar{P})\right)$, then $\operatorname{Split}_{p_{s}(\bar{P})}(t)=P_{t}$.

Define the following relations on $R$ :

$$
\begin{gathered}
\bar{P} \leq \bar{Q} \Leftrightarrow(\forall s) p_{s}(\bar{P}) \leq p_{s}(\bar{Q}), \\
\bar{P} \approx \bar{Q} \Leftrightarrow(\forall s) P_{s}={ }^{*} Q_{s} \wedge\left(\forall^{\infty} s\right) P_{s}=Q_{s}, \\
\bar{P} \leq^{*} \bar{Q} \Leftrightarrow\left(\exists \bar{P}^{\prime}\right) \bar{P} \approx \bar{P}^{\prime} \wedge \bar{P}^{\prime} \leq \bar{Q} .
\end{gathered}
$$

Fact 1.5 [GoJoSp, 4.14]. Assume $M A_{\kappa}(\sigma$-centered $)$. If $\left\langle\bar{P}_{\alpha}: \alpha\langle\kappa\rangle\right.$ is $a \leq^{*}$ decreasing sequence in $R$, then there exists $\bar{Q} \in R$ such that $(\forall \alpha<\kappa) \bar{Q} \leq^{*} \bar{P}_{\alpha}$.

The following two facts have proofs similar to those of Facts 1.3 and 1.4.

Fact 1.6. Suppose $X \in m^{0}$ and $\bar{P} \in R$. There exists $\bar{Q} \leq \bar{P}$ such that $(\forall s)\left[p_{s}(\bar{Q})\right] \cap X=\varnothing$.

Fact 1.7. Suppose $X \in m^{0}, \bar{P}, \bar{Q} \in R, \bar{P} \leq^{*} \bar{Q}$, and $(\forall s)\left[p_{s}(\bar{Q})\right] \cap X=\varnothing$. Then $(\forall s)\left[p_{s}(\bar{P})\right] \cap X=\varnothing$.

Now using, Facts 1.5, 1.6, 1.7 and the well-known result that for all $\kappa<\mathfrak{p}$ $M A_{\kappa}(\sigma$-centered) holds, a similar construction as in Theorem 1.1(1) shows that $\mathfrak{p} \leq \operatorname{add}\left(m^{0}\right)$.

\section{ADD AND COV ARE DISTINCT}

Definition 2.1. A set $A \subseteq{ }^{\omega} \omega$ is called strongly dominating if and only if

$$
\left(\forall f \in{ }^{\omega} \omega\right)(\exists \eta \in A)\left(\forall^{\infty} k\right) f(\eta(k-1))<\eta(k) .
$$

Definition 2.2. For any set $A \subseteq{ }^{\omega} \omega$, we define the domination game $D(A)$ as follows:

There are two players, GOOD and BAD. GOOD plays first. The game lasts $\omega$ moves.

\begin{tabular}{c|c} 
GOOD & BAD \\
\hline$s$ & $n_{0}$ \\
$m_{0}$ & $n_{1}$ \\
$m_{1}$ & \\
$\vdots$ & $\vdots$
\end{tabular}


The rules are: $s$ is a sequence in ${ }^{<\omega} \omega$, and the $n_{i}$ and $m_{i}$ are natural numbers. (Whoever breaks these rules first, loses immediately.)

The GOOD player wins if and only if:

(a) For all $i, m_{i}>n_{i}$.

(b) The sequence $s \frown m_{0} \frown m_{1} \frown \ldots$ is in $A$.

Lemma 2.3. Let $A \subseteq{ }^{\omega} \omega$ be a Borel set. Then the following are equivalent:

(1) There exists a Laver tree $p$ such that $[p] \subseteq A$.

(2) $A$ is strongly dominating.

(3) GOOD has a winning strategy in the game $D(A)$.

Remark. Strongly dominating is not the same as dominating. For example, the closed set

$$
A:=\left\{\eta \in{ }^{\omega} \omega:(\forall k) \eta(2 k)=\eta(2 k+1)\right\}
$$

is dominating but is not strongly dominating.

Proof of Lemma 2.3. We consider the following condition:

(4) (For all $F:<\omega \omega \times \omega \rightarrow \omega)(\exists \eta \in A)\left(\forall^{\infty} k\right)(\forall i \leq k) \eta(k)>F(\eta\lceil k, i)$. We will show $(1) \rightarrow(2) \rightarrow(4) \rightarrow(3) \rightarrow(1)$.

$(1) \rightarrow(2)$ is clear.

$(2) \rightarrow(4)$ : Given $F$, define $f$ by

$$
f(m):=\max \left\{F(s, i): i \leq m, s \in m^{\leq m+1}\right\}+m
$$

$f$ is increasing, $f(m) \geq m$ for all $m$.

Find $\eta$ such that $\left(\forall^{\infty} k\right) \eta(k)>f(\eta(k-1))$. Then $\eta$ is increasing. For almost all $k$ we have, letting $m:=\eta(k-1): m \geq k-1$, so $\eta \uparrow k \in m^{\leq m+1}$, so by the definition of $f$ we get $f(m) \geq F(\eta\lceil k, i)$ for any $i \leq k$. So $\eta(k)>f(\eta(k-1) \geq F(\eta\lceil k, i)$.

$(4) \rightarrow(3)$ : Assume that GOOD has no winning strategy. Then BAD has a winning strategy $\sigma$ (since the game $D(A)$ is Borel, hence determined).

We can find a function $F:{ }^{<\omega} \omega \times \omega \rightarrow \omega$ such that for all $s, m_{0}, \ldots, m_{k}$ we have

$$
\sigma\left(s, m_{0}, \ldots, m_{k}\right)=F\left(s \frown m_{0} \frown \ldots \frown m_{k},|s|\right) .
$$

Find $\eta \in A$ as in (4). So there is $k_{0}$ such that $\forall k \geq k_{0} \quad \eta(k) \geq F\left(\eta \uparrow k, k_{0}\right)$. So in the play

\begin{tabular}{c|c} 
GOOD & BAD \\
\hline$s:=\eta \uparrow k_{0}$ & $n_{0}:=\sigma(s)=F\left(\eta \uparrow k_{0}, k_{0}\right)$ \\
$m_{0}:=\eta\left(k_{0}+1\right)$ & $n_{1}:=\sigma\left(s, m_{0}\right)=F\left(\eta \uparrow\left(k_{0}+1\right), k_{0}\right)$ \\
$m_{1}:=\eta\left(k_{0}+2\right)$ & $\vdots$ \\
$\vdots$ & $\vdots$
\end{tabular}

player BAD followed the strategy $\sigma$, but player GOOD won, a contradiction.

$(3) \rightarrow(1)$ : Let $B$ be the set of all sequences $s \frown m_{0} \frown m_{1} \frown \ldots$ that can be played when GOOD follows a specific winning strategy. Clearly $B \subseteq A$, and for some Laver tree $p, B=[p]$. 
Lemma 2.4 [Ke]. Let $A \subseteq{ }^{\omega} \omega$ be an analytic set. Then the following are equivalent:

(1) There exists a Miller tree $p$ such that $[p] \subseteq A$.

(2) $A$ is unbounded in $\left({ }^{\omega} \omega, \leq^{*}\right)$.

Lemma 2.5. (1) Suppose $\mathfrak{b}=\mathfrak{c}$. For every dense open $D \subseteq \mathbb{L}$ there exists a maximal antichain $A \subseteq D$ such that

$$
\forall q \in \mathbb{L}\left([q] \subseteq \bigcup\{[p]: p \in A\} \Rightarrow \exists A^{\prime} \in[A]^{<c} \forall p \in A \backslash A^{\prime} p \perp q\right) .
$$

(2) The same is true for $\mathbb{M}$.

Proof. Let $\mathbb{L}=\left\{q_{\alpha}: \alpha<\mathfrak{c}\right\}$. Inductively we will define a set $S \subseteq \mathfrak{c}$ and sequences $\left\langle x_{\gamma}: \gamma\langle c\rangle\right.$ and $\left\langle p_{\gamma}: \gamma \in S\right\rangle$. Finally we will let $A=\left\{p_{\gamma}: \gamma \in S\right\}$.

Let $0 \in S$ and choose $x_{0} \in\left[q_{0}\right]$ arbitrarily.

It can easily be seen that every Laver tree contains $\mathfrak{c}$ extensions such that every two of them do not contain a common branch. So clearly we may find $p_{0} \in D$ such that $x_{0} \notin\left[p_{0}\right]$.

Now suppose that $\left\langle x_{\gamma}: \gamma\langle\alpha\rangle\right.$ and $\left\langle p_{\gamma}: \gamma \in S \cap \alpha\right\rangle$ have been constructed for $\alpha<\mathfrak{c}$.

First choose $x_{\alpha} \in\left[q_{\alpha}\right]$ arbitrarily, but such that, if $\left[q_{\alpha}\right] \nsubseteq \bigcup\left\{\left[p_{\gamma}\right]: \gamma<\alpha\right\}$, then $x_{\alpha} \notin \bigcup\left\{\left[p_{\gamma}\right]: \gamma<\alpha\right\}$.

In order to decide whether $\alpha \in S$ or not we distinguish the following two cases:

Case 1. $q_{\alpha}$ is compatible with some $p_{\gamma}, \gamma<\alpha$. In this case $\alpha \notin S$.

Case 2. $q_{\alpha}$ is incompatible with all $p_{\gamma}, \gamma<\alpha$. Now we let $\alpha \in S$, and we define $p_{\alpha}$ as follows:

By Lemma 2.3 for each $\gamma \in \alpha$ we may find $f_{\gamma}: \omega \rightarrow \omega$ such that

$$
\left(\forall \eta \in\left[p_{\gamma}\right] \cap\left[q_{\alpha}\right]\right)\left(\exists^{\infty} k\right) \eta(k) \leq f_{\gamma}(\eta(k-1)) .
$$

By our assumption on $\mathfrak{b}$ there exists a strictly increasing $f$ which dominates all the $f_{\gamma}^{\prime}$ 's. Now define $p_{\alpha}^{\prime} \in \mathbb{L}$ as follows: $\operatorname{stem}\left(p_{\alpha}^{\prime}\right)=\operatorname{stem}\left(q_{\alpha}\right)$, and for $t \in p_{\alpha}^{\prime}$, if $t \supseteq \operatorname{stem}\left(p_{\alpha}^{\prime}\right)$ and $|t|=: n$, we require

$$
\operatorname{Succ}_{p_{\alpha}^{\prime}}(t)=\operatorname{Succ}_{q_{\alpha}}(t) \cap[f(t(n-1)), \infty) .
$$

Clearly $p_{\alpha}^{\prime} \in \mathbb{L}, p_{\alpha}^{\prime} \subseteq q_{\alpha}$, and by (**) and our assumption on $f$ we conclude $\left[p_{\gamma}\right] \cap\left[p_{\alpha}^{\prime}\right]=\varnothing$ for every $\gamma<\alpha$.

By the remark above that every Laver tree contains $c$ extensions such that every two of them do not contain a common branch, we may find $p_{\alpha} \in D$ such that $p_{\alpha}$ extends $p_{\alpha}^{\prime}$ and $\left[p_{\alpha}\right]$ and $\left\{x_{\gamma}: \gamma \leq \alpha\right\}$ are disjoint.

This finishes the construction. Now let $A:=\left\{p_{\gamma}: \gamma \in S\right\}$.

Since every $q_{\alpha}$ is either compatible with some $p_{\gamma}, \gamma<\alpha$ (Case 1) or contains the condition $p_{\alpha}$ (Case 2), and for $\alpha \neq \gamma$ with $\alpha, \gamma \in S$ we have $\left[p_{\alpha}\right] \cap\left[p_{\gamma}\right]=$ $\varnothing$, we conclude that $A$ is a maximal antichain.

$A$ also satisfies condition $(*)$ : Let $q=q_{\alpha}$. By construction, if $\left[q_{\alpha}\right] \nsubseteq$ $\bigcup\left\{\left[p_{\gamma}\right]: \gamma \in S \cap \alpha\right\}$, then $\left[q_{\alpha}\right] \nsubseteq \bigcup\left\{\left[p_{\gamma}\right]: \gamma \in S\right\}$.

The proof of (2) is analogous, but instead of Lemma 2.3 we use Lemma 2.4.

Lemma 2.6. Suppose $\mathfrak{b}=\mathfrak{c}$. Then $\operatorname{add}\left(l^{0}\right) \leq \kappa(\mathbb{L})$ and $\operatorname{add}\left(m^{0}\right) \leq \kappa(\mathbb{M})$. 
Proof. We may assume $\kappa(\mathbb{L})<\mathfrak{c}$. Let $\dot{f}$ be a $\mathbb{L}$-name such that $\mathbb{}_{\mathbb{L}}$ " $\dot{f}$ : $\kappa(\mathbb{L}) \rightarrow \mathfrak{c}$ is onto". For $\alpha<\kappa(\mathbb{L})$ let

$$
D_{\alpha}:=\left\{p \in \mathbb{L}:(\exists \beta) p \Vdash_{\mathbb{L}} \dot{f}(\alpha)=\beta\right\} .
$$

For $p \in D_{\alpha}$ we write $\beta_{p}=\beta_{p}(\alpha)$ for the unique $\beta$ satisfying $p \Vdash_{\mathbb{L}} \dot{f}(\alpha)=\beta$.

Clearly $D_{\alpha}$ is dense and open. So we may choose a maximal antichain $A_{\alpha} \subseteq D_{\alpha}$ as in Lemma 2.5. Let

$$
X_{\alpha}:={ }^{\omega} \omega \backslash \bigcup\left\{[p]: p \in A_{\alpha}\right\} .
$$

Then $X_{\alpha} \in l^{0}$. We claim that $X=\bigcup_{\alpha<\kappa(\mathbb{L})} X_{\alpha} \notin l^{0}$. Suppose on the contrary $X \in l^{0}$. So we may find $q \in \mathbb{L}$ such that $[q] \cap X=\varnothing$ and hence $[q] \subseteq \bigcup\{[p]$ : $\left.p \in A_{\alpha}\right\}$ for each $\alpha$. By the choice of $A_{\alpha}$ each of the sets

$$
B_{\alpha}:=\left\{\beta_{p}(\alpha): p \in A_{\alpha}, p \text { compatible with } q\right\}
$$

is bounded in $\mathfrak{c}$. Since $\mathfrak{c}$ is regular by our assumption $\mathfrak{b}=\mathfrak{c}$, we can find $\nu<\mathfrak{c}$ such that for all $\alpha<\kappa(\mathbb{L}), B_{\alpha} \subseteq \nu$. So easily conclude that

$$
q \Vdash_{\mathbb{L}} \text { "ran }(\dot{f}) \subseteq \nu<c " .
$$

This is a contradiction.

The proof for $\mathbb{M}$ is similar.

Theorem 2.7. $\kappa(\mathbb{L}) \leq \mathfrak{h}$ and $\kappa(\mathbb{M}) \leq \mathfrak{h}$.

Proof. We prove it only for $\mathbb{L}$. The proof for $\mathbb{M}$ is very similar. We work in $V$. Let $\left\langle\mathscr{A}_{\alpha}: \alpha<\mathfrak{h}\right\rangle$ be a family of maximal almost disjoint families such that:

(1) if $\alpha<\beta<\mathfrak{c}$, then $\mathscr{A}_{\beta}$ refines $\mathscr{A}_{\alpha}$;

(2) there exists no maximal almost disjoint family refining all the $\mathscr{A}_{\alpha}$;

(3) $\bigcup\left\{\mathscr{A}_{\alpha}: \alpha<\mathfrak{h}\right\}$ is dense in $\left([\omega]^{\omega}, \subseteq^{*}\right)$.

That such a sequence exists was shown in [BaPeSi].

Since $\mathfrak{h}$ is regular, for every $p \in \mathbb{L}$ there exists $\alpha<\mathfrak{h}$ such that for each $s \in \operatorname{Split}(p)$ there is $A \in \mathscr{A}_{\alpha}$ with $A \subseteq^{*} \operatorname{Succ}_{p}(s)$. Hence, writing $\mathbb{L}_{\alpha}$ for the set of those $p \in \mathbb{L}$ for which $\alpha$ has the property just stated, we conclude $\mathbb{L}=\bigcup\left\{\mathbb{L}_{\alpha}: \alpha<\mathfrak{h}\right\}$.

For each $A \in \mathscr{A}_{\alpha}$ choose $\mathscr{B}_{A}=\left\{B^{A}(p): p \in \mathbb{L}\right\}$, a maximal almost disjoint family on $A$.

Now we will define $\mathbb{L}_{\alpha}^{\prime}:=\left\{q^{\alpha}(p): p \in \mathbb{L}_{\alpha}\right\}$ such that $q^{\alpha}(p)$ extends $p$ for every $p \in \mathbb{L}_{\alpha}$ and $p_{1} \neq p_{2}$ implies $q^{\alpha}\left(p_{1}\right) \perp q^{\alpha}\left(p_{2}\right)$. For $p \in \mathbb{L}_{\alpha}, q^{\alpha}(p)$ will be defined as follows:

For each $s \in \operatorname{Split}(p)$ let $C_{s}^{\alpha}(p):=\operatorname{Succ}_{p}(s) \cap B^{A}(p)$ where $A \in \mathscr{A}_{\alpha}$ is such that $A \subseteq^{*} \operatorname{Succ}_{p}(s)$. So clearly $C_{s}^{\alpha}(p)$ is infinite. Now $q^{\alpha}(p)$ is the unique Laver tree $\leq p$ satisfying $\operatorname{stem}\left(q^{\alpha}(p)\right)=\operatorname{stem}(p)$ and for each $s \in \operatorname{Split}\left(q^{\alpha}(p)\right)$ we have $\operatorname{Succ}_{q^{\alpha}(p)}(s)=C_{s}^{\alpha}(p)$.

It is not difficult to see that $\mathbb{L}_{\alpha}^{\prime}$ has the stated properties.

Now we are ready to define a $\mathbb{L}$-name $\dot{f}$ such that $\Vdash_{\mathbb{L}}$ " $\dot{f}: \mathfrak{h}^{V} \rightarrow \mathfrak{c}^{V}$ is onto": For each $p \in \mathbb{L}_{\alpha}$, let $\left\{r_{\xi}^{\alpha}(p): \xi<\mathfrak{c}\right\} \subseteq \mathbb{L}$ be a maximal antichain below $q^{\alpha}(p)$, and define $\dot{f}$ in such a way that $r_{\xi}^{\alpha}(p) \Vdash_{\mathbb{L}}$ " $\dot{f}(\alpha)=\xi$ ". As $\bigcup\left\{\mathbb{L}_{\alpha}^{\prime}: \alpha<\mathfrak{h}\right\}$ is dense in $\mathbb{L}$, it is easy to check that $\dot{f}$ is as desired. 
Theorem 2.8. Let $\omega_{2}=S_{\mathrm{M}} \dot{\cup} S_{\mathrm{L}}$, where the sets $S_{\mathrm{M}}$ and $S_{\mathrm{L}}$ are disjoint and stationary. Let $\left(P_{\alpha}, Q_{\alpha}: \alpha<\omega_{2}\right)$ be a countable support iteration of length $\omega_{2}$ such that for all $\alpha$ we have $\Vdash_{P_{\alpha}} Q_{\alpha}=\mathbb{M}$ whenever $\alpha \in S_{\mathbf{M}}$, and $\Vdash_{P_{\alpha}} Q_{\alpha}=\mathbb{L}$ otherwise. Also suppose that $V$ satisfies $C H$. Then in $V^{P}, \mathfrak{h}=\omega_{1}$ holds.

Proof. Both $\mathbb{M}$ and $\mathbb{L}$ have the property $(*)_{1}$ of [JuSh]. (For $\mathbb{L}$, this was proved in [JuSh] and for $\mathbb{M}$ this was proved in [BaJuSh].) [JuSh] also showed that this property is preserved under countable support iterations, so also $P_{\omega_{2}}$ has this property. Hence, the reals of $V$ do not have measure zero in $V^{P}$, so from $\mathfrak{h} \leq \mathfrak{s} \leq \operatorname{unif}(\mathfrak{L}$ ) (where $\mathfrak{s}$ is the splitting number and unif( $\mathfrak{L}$ ) is the smallest cardinality of a set of reals which is not null) we get the desired conclusion.

Theorem 2.9. Let $P_{\omega_{2}}$ be as in Theorem 2.8. Then

$$
V^{P_{\omega_{2}}}=\omega_{1}=\operatorname{add}\left(l^{0}\right)=\operatorname{add}\left(m^{0}\right)<\operatorname{cov}\left(l^{0}\right)=\operatorname{cov}\left(m^{0}\right)=\omega_{2} .
$$

Proof. Since $\mathbb{L}$ adds a dominating real, we have $V^{P_{\omega_{2}}} \models \mathfrak{b}=\mathfrak{c}$; so by Lemma 2.6 and Theorems 2.7 and 2.8 it suffices to prove that the covering coefficients are $\omega_{2}$ in the respective models. The proof of this is similar to the proof of [JuMiSh, Theorem 1.2] that cov of the Marczewski ideal is $\omega_{2}$ in the iterated Sacks's forcing model.

We give the proof only for $l^{0}$. Suppose $\left\langle X_{\alpha}: \alpha<\omega_{1}\right\rangle \in V^{P_{\omega_{2}}}$ is a sequence of $l^{0}$-sets. In $V^{P_{\omega_{2}}}$ let $f_{\alpha}: \mathbb{L} \rightarrow \mathbb{L}$ be such that for every $p \in \mathbb{L}, f_{\alpha}(p)$ extends $p$ and $\left[f_{\alpha}(p)\right] \cap X_{\alpha}=\varnothing$. Since $P_{\omega_{2}}$ has the $\omega_{2}$-chain condition, by a Löwenheim-Skolem argument it is possible to find $\gamma<\omega_{2}$ such that

$$
\left\langle f_{\alpha} \mid \mathbb{L}^{V_{y}}: \alpha<\omega_{1}\right\rangle \in V^{P_{y}}
$$

where $V_{\gamma}:=V^{P_{y}}$. Moreover, it is possible to find such a $\gamma$ in $S_{\mathrm{L}}$. We claim that the Laver real $x_{\gamma}$ (which is added by $Q_{\gamma}=\mathbb{L}^{V_{y}}$ ) is not in $\bigcup_{\alpha<\omega_{1}} X_{\alpha}$, which will finish the proof. Otherwise, for some $p \in \mathbb{L}_{\gamma \omega_{2}}$ where $\mathbb{L}_{\gamma \omega_{2}}:=\mathbb{L}_{\omega_{2}} / G_{\gamma}$ and some $\alpha<\omega_{1}$ we would have $p \Vdash x_{\gamma} \in X_{\alpha}$. But letting $q:=p(\gamma) \in \mathbb{L}$ and letting $r(\gamma):=f_{\alpha}(q)$ and $r(\beta):=p(\beta)$ for $\beta>\gamma$ we see that $r \Vdash x_{\gamma} \notin X_{\alpha}$, a contradiction.

\section{REFERENCES}

[BaJuSh] T. Bartoszynski, H. Judah, and S. Shelah, Cichon's diagram, J. Symbolic Logic 58 (1993), 401-423.

[BaPeSi] B. Balcar, J. Pelant, and P. Simon, The space of ultrafilters on $N$ covered by nowhere dense sets, Fund. Math. 110 (1980), 11-24.

[BaLa] J. E. Baumgartner and R. Laver, Iterated perfect set forcing, Ann. Math. Logic 17 (1979), 271-288.

[GoJoSp] M. Goldstern, M. Johnson, and O. Spinas, Towers on trees, Proc. Amer. Math. Soc. 122 (1994), 557-564.

[JuMiSh] H. Judah, A. Miller, and S. Shelah, Sacks forcing, Laver forcing and Martin's axiom, Arch. Math. Logic 31 (1992), 145-161.

[JuSh] H. Judah and S. Shelah, The Kunen-Miller chart, J. Symbolic Logic 55 (1990), 909-927.

[Ke] A. Kechris, A notion of smallness for subsets of the Baire space, Trans. Amer. Math. Soc. 229 (1977), 191-207.

[La] R. Laver, On the consistency of Borel's conjecture, Acta Math. 137 (1976), 151-169. 
[Mi] A. Miller, Rational perfect set forcing, Contemp. Math. (J. E. Baumgartner, D. Martin, and S. Shelah, eds.), vol. 31, Amer. Math. Soc., Providence, RI, 1984, pp. 143-159.

[P1] S. Plewik, On completely Ramsey sets, Fund. Math. 127 (1986), 127-132.

2. Mathematisches Institut, Freie Universität Berlin, ARnimallee 3, 14195 Berlin, GeRMANY

E-mail address: goldstrn@math.fu-berlin.de

MatematickÝ ústav SAV, Jesenná 5, 04154 Košice, Slovakia

E-mail address: repicky@kosice.upjs.sk

InStitute of Mathematics, Hebrew University of JeRUSALEM, JeRUSALEM, ISRAEL

E-mail address: shelah@math.huji.ac.il

Departement Mathematik, ETH-Zentrum, 8092 ZÜrich, Switzerland

Current address: Department of Mathematics, University of California, Irvine, California 92717 